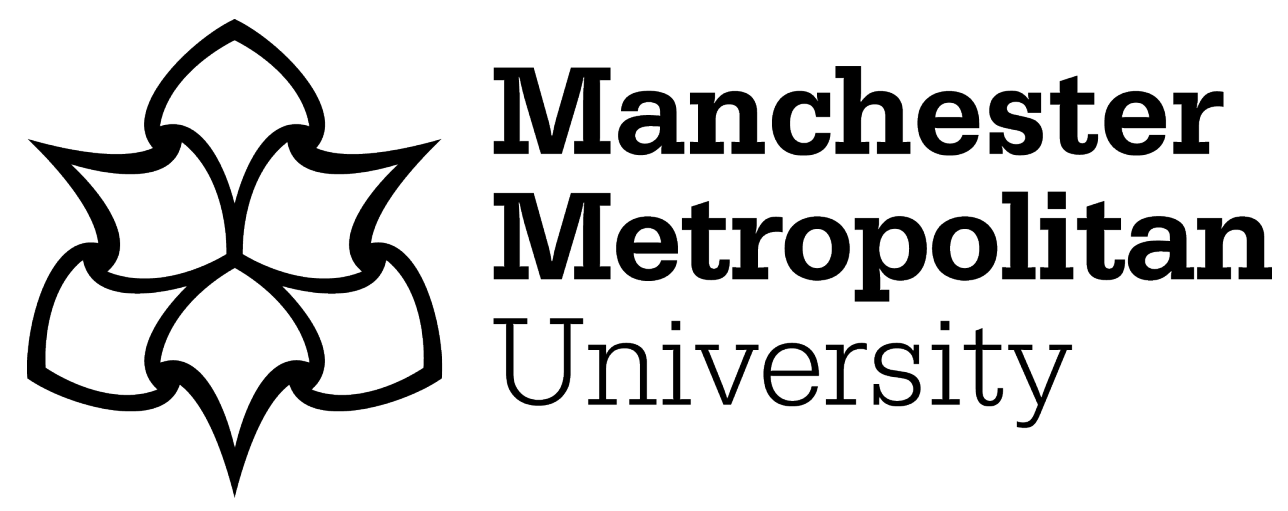

Collins, T, Woolley, SI, Rawson, NC and Haroon, L (2016) Final-year projects using open source OpenEEG. Computer Applications in Engineering Education, 24 (1). pp. 156-164. ISSN 1061-3773

Downloaded from: https://e-space.mmu.ac.uk/874/

Version: Accepted Version

Publisher: Wiley

DOI: https://doi.org/10.1002/cae.21682

Please cite the published version 


\title{
Final-year projects using open source OpenEEG
}

\author{
Tim Collins, Sandra I. Woolley, Nicholas C. Rawson and Luth Haroon
}

\begin{abstract}
This paper describes undergraduate electroencephalography projects using the OpenEEG platform. The projects applied a broad range of curriculum components including computer interfacing, signal processing and software development. Project applications were wide-ranging and provided opportunities to explore research areas such as Brain-Computer Interfacing, affective computing and assistive technology.
\end{abstract}

Index Terms - Electroencephalography EEG, OpenEEG, Brain-Computer Interfacing BCl, Final-Year Project, Open Source.

\section{INTRODUCTION}

OpenEEG [1] is an open source community hardware and software resource for low-cost electroencephalography (EEG). The OpenEEG software and hardware are released under a variety of free licenses. The Modular OpenEEG design is the most popular design used by OpenEEG developers and is supported by a range of documentation and a software development forum.

Modular OpenEEG devices were used for final-year EEG project themes involving aspects of signal processing and computer interfacing. The project applications ranged from gaming to health and provided opportunities to explore research areas such as affective computing, pervasive health and Brain-Computer Interfacing (BCI). The EEG project theme proved popular with students on consecutive years and provided scope for a broad range of individual projects.

\section{BACKGROUND}

Some examples of EEG activities and projects in undergraduate electronic engineering education are reported in the literature. As would be anticipated, laboratory activities involving physiological signals are more commonly associated with biomedical engineering programs, where students may expect to perform exercises involving the acquisition and filtering of a variety of physiological signals with proprietary hardware and software [2]. Electronic engineering undergraduates with opportunities to take biomedical engineering modules might expect similar opportunities to work with commercial equipment. Rhodes and Dune [3] outline a bioelectric potentials module for electronic engineering majors incorporating ECG (electrocardiography) amplifier and wave deflection detector circuit construction activities, and the measurement of their own ECG, EEG and EMG (electromyography, muscle signal) signals using the ADInstruments PTB 32 Teaching System.

Lee et al. [4] report the use of EEG signals in a first year electronic engineering laboratory as examples of real, noisy, low amplitude signals with which to explore operational amplifiers, analog filters and sensor interface circuitry. Scolaro et al. [5] describe the design of an in-house 6-channel, ARM-based acquisition system designed for electronic engineering students performing biomedical engineering laboratory exercises with wireless electrodes for EEG, ECG, EMG and EOG (electrooculography: eye movement signals). The aim of the exercise was to familiarize students with aspects of signal acquisition, characteristics and filtering. Jain and Gluckman [6] report on the design of an 8-channel EEG system based on Texas Instruments' biopotential amplifier and 16-bit microcontroller. The system was designed as a non-invasive $\mathrm{BCI}$ for teaching or research.

The taught components of undergraduate degree electronic engineering programs at the University of Birmingham do not use EEG equipment. However, relevant underlying theory and technological 
components are taught in the form of analog and digital electronics, embedded systems and digital signal processing. Final-year projects involving physiological signal inputs would typically require significant effort on lower-level design and development, e.g., circuit design and construction, and achieving the basic signal interface. Modular OpenEEG open source hardware and software provided an interesting opportunity to resource final-year projects for the capture of EEG signals so that there could be more scope to focus on higher-level design and development. For example, students could invest more effort in implementing ideas relevant to research and the real-world, and more effort in signal processing, application design and experimental testing. In this way, the OpenEEG project themes provided an opportunity to bring together a selection of topics from the electronic engineering curriculum and place them in the context of current research and real-world applications. Additionally, OpenEEG provided an opportunity to achieve this at a modest cost.

In recent years, open source software has received increased interest for educational applications, primarily on financial grounds but also due to the possibility of customization [7]. It has been noted, however, that the adoption of open source software is not entirely without cost [8]. The often sparse documentation and lack of customer support should be accounted for when considering open source solutions. Despite this, it is believed that the benefits of open source software for education generally outweigh the costs [8].

There is limited documentation regarding the use of OpenEEG in academic project work. Brainathlon [9] is a Modular OpenEEG application developed as part of a master's project. It is a game for one or two players that provides visualizations of selected frequencies which players can attempt to control. The Brainathlon application is one of the software resources available on the OpenEEG website for download. Another example of student work is BrainBay [10], it is also a software resource application on the OpenEEG website. It was designed as a brain-computer interface for people with limited physical function.

EEG signals. EEG signals can be acquired across the scalp. The International10-20 system [11] defines a set of 19 locations, shown in Fig. 1., for EEG electrode placement. The number of electrodes required, however, is much reduced by knowing the location of signals of interest.

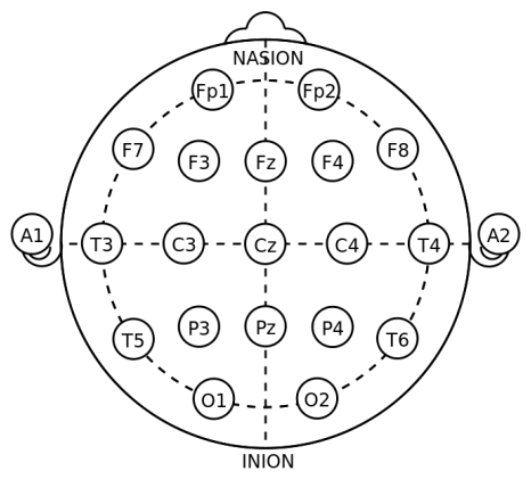

Fig. 1. The EEG electrode locations defined by the $10-20$ system.

(The labels F, T, C, P and $\mathrm{O}$ denote frontal, temporal, central, parietal, and occipital lobes, respectively. Nasion is the point above the bridge of the nose and inion is the most prominent point at the back of the head.)

EEG signal analysis can be performed in either the frequency domain or in the time domain. In the frequency domain, as shown in table I, lower frequencies tend to be associated with less active states and higher frequencies with more alert and active states. So, for example, an EEG relaxation 
application may involve feeding back information about higher and lower frequency bands, encouraging the increase of a lower band of frequencies and the decrease of a higher band of frequencies.

TABLE I

\begin{tabular}{ccl}
\multicolumn{2}{c}{ EEG FREQUENCIES AND EXAMPLES OF BROADLY ASSOCIATED STATES [9] } \\
\hline Type & $\begin{array}{c}\text { Frequency } \\
\text { Range (Hz) }\end{array}$ & \multicolumn{1}{c}{ Example Associations } \\
\hline Delta & up to 4 & $\begin{array}{l}\text { Normal for infants. Associated with sleep phases in } \\
\text { adults. }\end{array}$ \\
\hline Theta & $4-8$ & $\begin{array}{l}\text { Normal in children and in sleep. Associated with } \\
\text { hypnagogic states (waking up/falling asleep) and } \\
\text { low-levels of alertness. Elevated, together with } \\
\text { alpha, associated with meditation. }\end{array}$ \\
\hline Alpha & $8-13$ & $\begin{array}{l}\text { Associated with relaxed and reflecting states. Eyes } \\
\text { closed. Also associated with inhibition control. }\end{array}$ \\
\hline Beta & approx.13-30 & Associated with active and alert states. Eyes open. \\
\hline Gamma & $30-100+$ & Associated with higher cognitive processes. \\
\hline Mu & $8-13$ & $\begin{array}{l}\text { Sensorimotor rhythms of the motor cortex. } \\
\text { Associated with voluntary movement }\end{array}$ \\
\hline \hline
\end{tabular}

An example of an EEG time domain feature of potential interest is the P300 wave. The P300 wave is an event-related potential, that is, a brain response to an event such as a sensory, cognitive or motor event. It is generated approximately $300 \mathrm{~ms}$ after an event and has been used in brain computer interfacing (BCl). The P300 speller [12-14], is an example of a P300 BCl application where subjects generate P300 waves when a letter matching their choice is illuminated on a screen in front of them. In this way subjects can compose, edit and send messages. Improving the performance of classification of the P300 waves can result in high accuracy message generation [15]. Another example of a P300 $\mathrm{BCl}$ application is, 'Brain-Painting', a paint program controlled by the P300 waves of people with amyotrophic lateral sclerosis (ALS), a degenerative disease of progressive paralysis [16]. Similar applications in assistive technology include, for example, $\mathrm{BCl}$ interfaces for wheelchair control [17].

\section{UNDERGRADUATE PROJECTS}

Final-year masters (year 4 M.Eng.) and bachelors (year 3 B.Eng.) students were presented with project outlines and opportunities to consult with potential supervisors. Although the students had no prior experience of EEG, the EEG project themes proved popular and there were many requests from the cohort. Students reported varying reasons for their selections, for example, expressing interests in creating EEG game interfaces and interests in health applications. The projects were seen to provide a range of practical and academic opportunities with tangible experimental components from which data could be acquired and analyzed, and conclusions made.

Students were encouraged to explore connections with relevant research areas such as rehabilitation and assistive technology, and affective computing, and to consider potential applications, for example, communication mechanisms for locked-in patients. Some of these findings, together with a variety of pointers, links and notes were shared on a project blog. Other files and resources were shared in an on-line repository (Dropbox [18]).

\section{OPENEEG}

The Modular OpenEEG design comprises an analog amplifier board and a digital signal capture board. The basic design supports 2 EEG channels. With the addition of one or two more analog boards, it can 
expand to four or six channels, respectively. Two channels were sufficient for the projects, but the option to expand was desirable.

Populated EEG boards were purchased from Olimex [19], a European electronics supplier of populated and unpopulated PCBs of the Modular OpenEEG design. At the time of writing, the populated analog and digital boards cost approximately $\$ 80$ and $\$ 53$, respectively. Electrodes, cables, connectors and housings were purchased separately. Abrasive skin prepping gel and conductive EEG paste were also required. This made the final cost of each complete system approximately $\$ 300$.

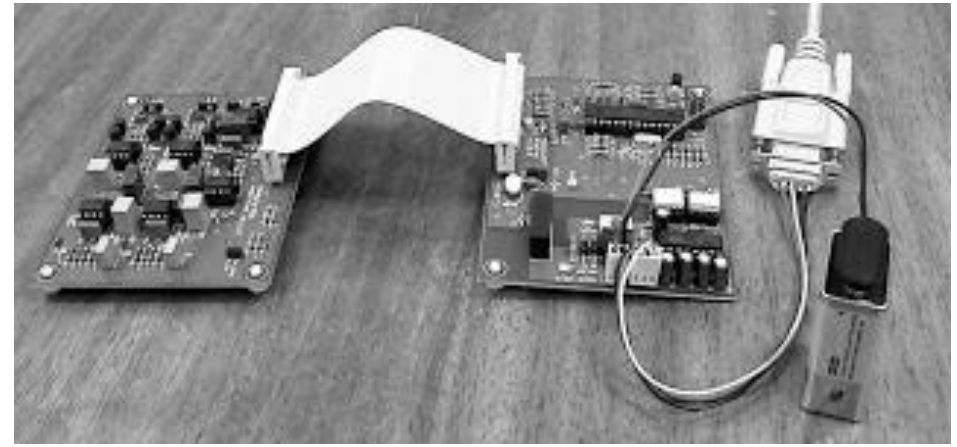

Fig. 2. The Olimex Modular OpenEEG analog and digital boards [19].

Fig. 2 shows the Olimex analog and digital boards. The analog board amplifies and filters the microvolt EEG signal and a right-leg driver reduces common mode interference, primarily from $50 \mathrm{~Hz} \mathrm{UK}(60 \mathrm{~Hz}$ USA) mains hum, by subtraction. The digital board uses the Atmel ATmega8 microcontroller and outputs 6 channel EEG packets via an RS-232 interface. This caused some difficulties for students who wanted to use the equipment with laptops which did not have RS-232 ports. Some reliability problems with USB-to-RS-232 adaptors exacerbated troubleshooting when acquiring signals. However, the onboard Modular OpenEEG $14 \mathrm{~Hz}$ square wave calibration signal proved useful for testing communications.

Health and Safety, and Ethical Approval: In order to detect EEG signals, electrodes need to make contact with the skin surface of the subject. For this reason, electrical safety is a critical characteristic of the circuits. The digital board provides electrical isolation from the communications port and from the power supply, however, as an additional safety measure, it was stipulated in the projects that all experiments were battery powered. In addition to health and safety issues, the projects provided an opportunity to consider a number of ethical issues, primarily arising from the use of human subjects.

Students completed the systems themselves by making up connecting cables and, later, housing the amplifier board in metal enclosures. Passive, unshielded $10 \mathrm{~mm}$ silver chloride disc electrodes were used. With practice, the careful use of abrasive skin preparation gel and application of conductive paste improved secure scalp positioning and led to much better signal acquisition. Shielded electrode cabling was investigated but the lighter-weight unshielded cabling proved adequate and made securing electrodes easier.

\section{COMPUTER INTERFACING: The Modular OpenEEG Data Format}

The modular OpenEEG digital boards output 256 EEG samples from the 6 possible channels per second in a "Firmware 2" format (as defined in the Modular EEG "Firmware 2" [1]). The samples are signed 10-bit values stored as 2 byte words (such that the upper 6 bits of the MSB are unused). Each sample is contained within a 17 byte data frame which has a 4 byte header (comprising a two byte synchronization value, a version byte and a frame number byte), 12 bytes of EEG values ( 2 bytes for each of 6 channels) and followed by a single (unused) button state byte (allowing the connection of four external buttons or switches should an application require them). 
TABLE II

The 17-Byte DATA Frame OF MODULAR OPENEEG FIRMWARE 2 DATA FORMAT [1]

\begin{tabular}{l}
\hline Byte 1: Sync Value 0xa5 \\
\hline Byte 2: Sync Value 0x5a \\
\hline Byte 3: Version (2) \\
\hline Byte 4: Frame Number \\
\hline Byte 5: Channel 1 High Byte \\
\hline Byte 6: Channel 1 Low Byte \\
\hline Byte 7: Channel 2 High Byte \\
\hline Byte 8: Channel 2 Low Byte \\
\hline Byte 9: Channel 3 High Byte \\
\hline Byte 10: Channel 3 Low Byte \\
\hline Byte 11: Channel 4 High Byte \\
\hline Byte 12: Channel 4 Low Byte \\
\hline Byte 13: Channel 5 High Byte \\
\hline Byte 14: Channel 5 Low Byte \\
\hline Byte 15: Channel 6 High Byte \\
\hline Byte 16: Channel 6 Low Byte \\
\hline Byte 17: Button States (b1-b4) \\
\hline \hline
\end{tabular}

\section{A. Code Resources}

\section{SUPPORTING RESOURCES}

OpenEEG provides a number of open source and closed source applications for biofeedback and EEG analysis. The project students were required to develop their own applications; however, the various example applications were useful in terms of exploring alternative approaches and visualizations.

.NET libraries and VB, C\# and MATLAB [20] code examples were prepared by the supervisors and provided to the students to help them get started, test their hardware and capture signals. For example, a .NET signal acquisition library was written to enable students to capture signals outputted by the digital board. This library handled frame synchronization and data formatting, presenting the incoming samples to the host application as objects containing six, sixteen-bit integers. Students could then use either the VB or C\# example code to acquire and display EEG data in real-time.

Most students made some use of the example code though, for example, one student chose to write code independently in Python. Importantly, use of any provided code was made explicit in all submitted work. The example code supplied to the students is available on-line at http://www.eee.bham.ac.uk/collinst/eeg.html.

To help students with spectrum analysis implementation, further example code was provided. As shown in Fig. 3, this code displays both time and frequency domain plots of the incoming EEG data in realtime. This code makes use of the open source Exocortex .NET FFT library [21]. 


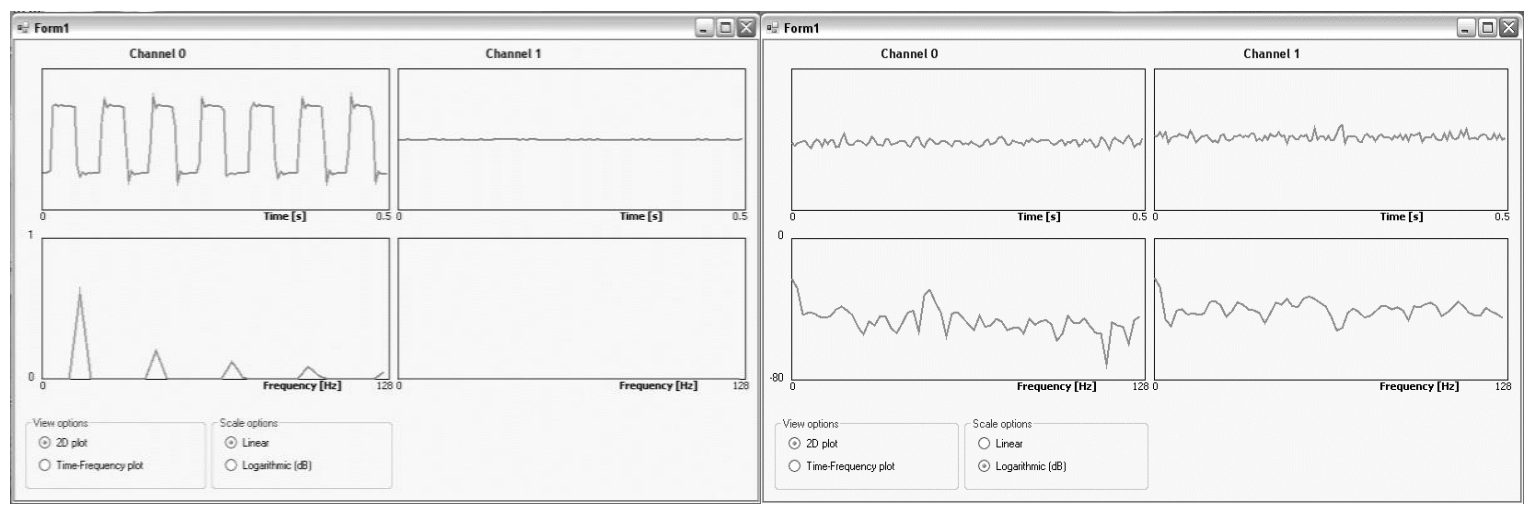

a)

b)

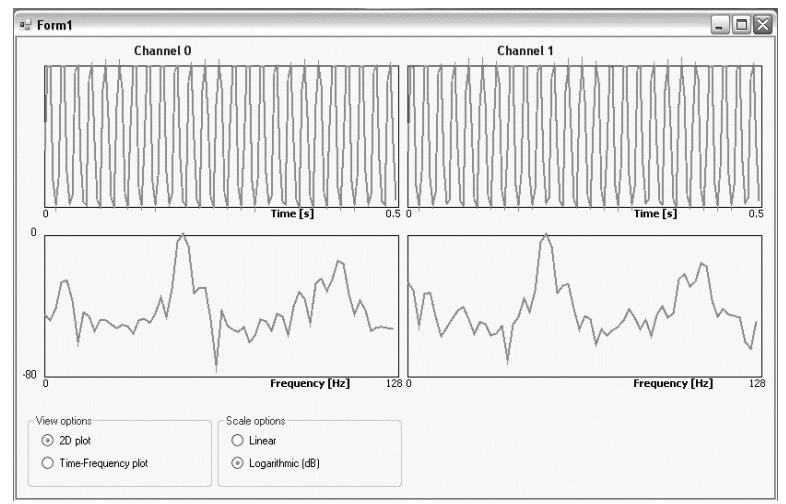

c)

Fig. 3. Example screenshots of the output of C\# sample code using the .NET library provided for students. In each window, Channel 0 is shown on the left and Channel 1 on the right. The top plot displays the signal in the time-domain over a $0.5 \mathrm{~s}$ duration and the plot below is the corresponding spectral analysis from 0-128 Hz. a) Shows the Calibration signal on Channel 0 and no input to Channel 1. b) Shows inputs to both Channels 0 and 1. c) Shows inputs to both Channels 0 and 1 without the right leg driver causing them to be badly affected by interference, in this case mains hum.

Documented example MATLAB and C\# code for a simple short-time (typically 0.5-1 s) Fourier Transform was also provided for the students. An example of a Hamming windowed Fourier Transform output as a MATLAB colormap is shown in the blog post in Fig. 4. Again the code is available at http://www.eee.bham.ac.uk/collinst/eeg.html.

Most of the project specifications required students to perform a time-frequency analysis of the EEG data in order to evaluate the relative activity levels in the EEG frequency bands described in Table I. This was most commonly achieved using either a Short-Time Fourier Transform (STFT) or a digital band-pass filter. When using the STFT, students needed to make design decisions about the observation length and the amplitude windowing used. Observation length influences the periodicity of the spectral analysis frames and is also inversely proportional to the frequency resolution of the transform. Different observation intervals were used according to which EEG bands needed to be resolved. Similar constraints affected the design of digital filters. As part of most of the projects, students were exposed to this kind of fundamental digital signal processing and, of course, there was scope for more sophisticated processing.

\section{B. The Project Blog}

A blog was created at the start of the projects as a resource to share useful information. Fig.4 shows a blog post about MATLAB time-frequency analysis and directs students to the Dropbox code repository. 


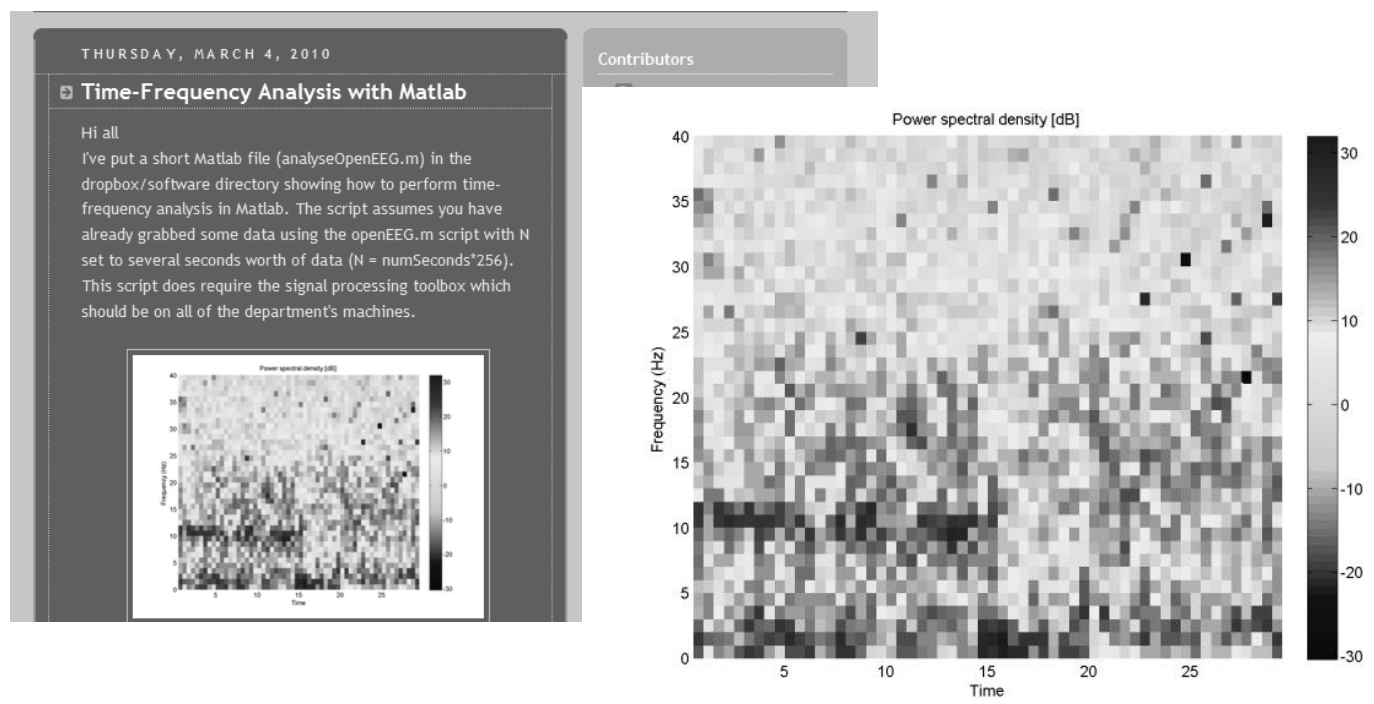

Fig. 4. The shared project blog post showing a MATLAB colormap Power Spectral Density

The students were added as blog authors and the blog was used to share background information and relevant technical information about the modular OpenEEG platform and supporting software.

\section{THE PROJECTS}

The undergraduate final-year EEG projects were offered in consecutive years. In total 7 B.Eng. and 5 M.Eng. projects were successfully completed. All students significantly contributed to the definition of their individual project brief with most expressing an interest in a particular direction or application at the outset. Several students had a keen interest in gaming and wanted to explore brain-computer interfacing while others were interested in health applications, whether for therapeutic support or for a communication or control means for people with limited physical motor capacity.

TABLE III. EXAMPLES OF PROJECT TITLES

\begin{tabular}{ll}
\hline \hline Project Title & Degree \\
\hline Neurofeedback Brain Training & B.Eng. \\
\hline Brain Powered Gaming Using Difficulty Level Control & B.Eng. \\
\hline Neurofeedback for Relaxation & B.Eng. \\
\hline Neurofeedback Relaxation Game & B.Eng. \\
\hline Neurofeedback as a Tool for Psychoacoustical Research & B.Eng. \\
\hline A P300 Based Brain Computer interface & B.Eng. \\
\hline A Brain-Controlled Computer Interface & B.Eng. \\
\hline Sensing the Motor Cortex & M.Eng. \\
\hline Visualization of Neural Activity & M.Eng. \\
\hline Mu Rhythm Analyzer & M.Eng. \\
\hline A P300 Speller & M.Eng. \\
\hline Neural Sensing with Therapeutic Feedback & M.Eng. \\
\hline \hline
\end{tabular}

As shown in Table III, the B.Eng. and M.Eng. projects involved a range of applications. The grades achieved in these projects spanned the range of typical outcomes. The EEG project B.Eng. average grade was slightly higher than the typical year average. 


\section{PROJECT EXAMPLES}

A. B.Eng. Project: Neurofeedback as a Tool for Psychoacoustical Research

The aim of the project was to investigate the effect of audio (in this case selected pieces of music) on the ability of the listener to increase theta-band frequencies associated with meditation.

The experimental design included a period of calibration before subjects listened to a random order selection of music (1. Indian Bhajan to Lord Shiva, 2. Tibetan Chant of Maitri Vihar Monastery, 3. Whirling Dervish (Mevlevi Order) Music, 4. Mozart's Requiem). As shown in Fig. 5., the animated opening of a flower was the mechanism for progress feedback. A closed flower indicating low thetaband activity and an open flower indicating higher theta-band activity.

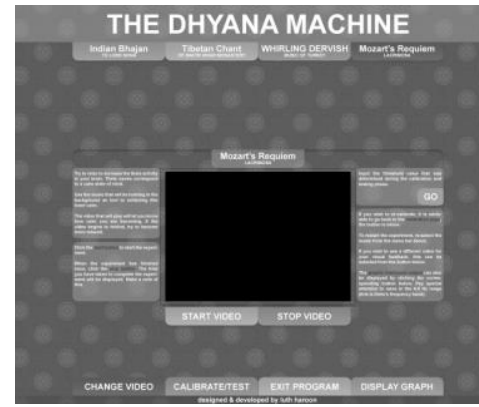

(a)

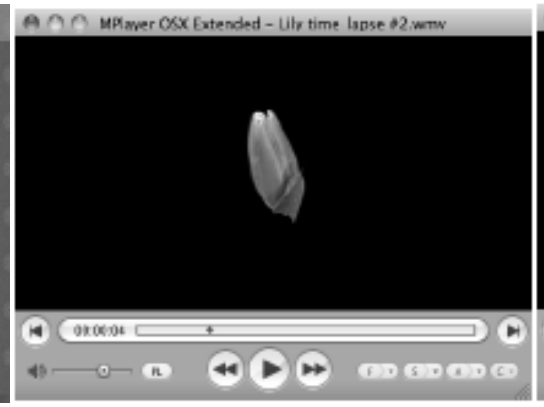

(b)

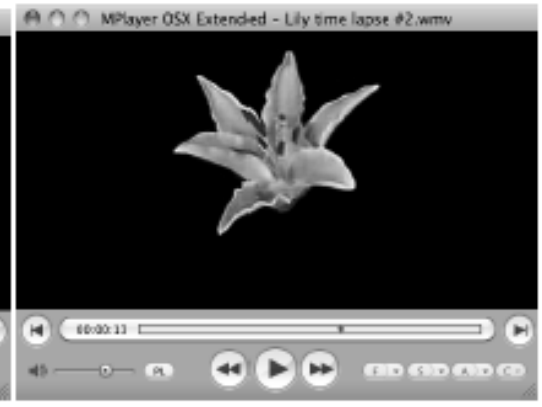

(c)

Fig. 5. (a) Application interface, and (b) and (c) output generated for low and high theta-band activity, respectively.

\section{B. An M.Eng. Project: Sensing the Motor Cortex}

The aim of the project was to research, develop and test a low-cost motor cortex detector and braincomputer interface $(\mathrm{BCl})$ that could be used to drive a game interface. This required the detection of motor imagery, i.e., periods when subjects think about moving. The secondary motor area (SMA), shown in Fig. 6., is involved in motor imagery [22]. The general function of the area is the planning and co-ordination of complex movements [23] this is in contrast to the primary motor cortex which is responsible for the execution of movement.

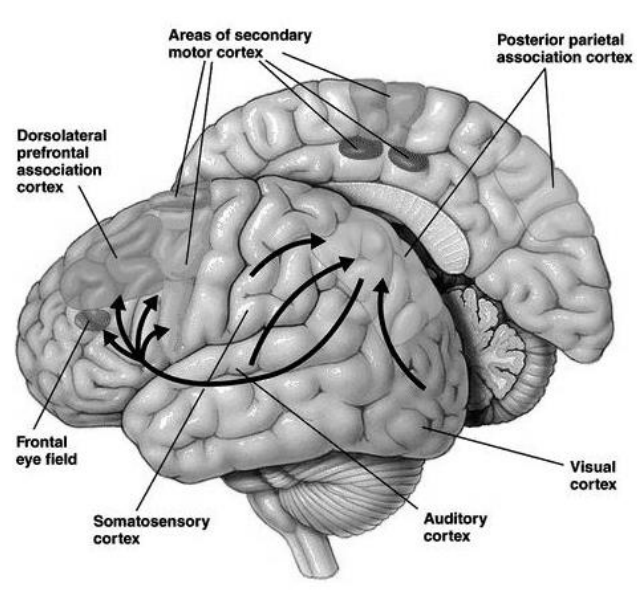

(a)

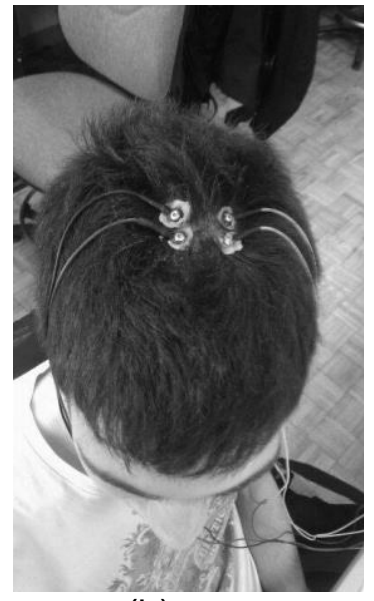

(b)

Fig. 6. Left, the areas of the secondary motor cortex and, right, the electrodes positioned for sensing activity in the area. 
The project required research into $\mathrm{BCls}$, and specifically $\mathrm{BCls}$ based on motor imagery. An experiment was designed that required test subjects to perform motor imagery tasks for specified times, interspersed with periods of no imagery.

\section{STUDENT FEEDBACK}

Students were asked to complete a short questionnaire about their EEG projects. The feedback provided is summarized in Table IV.

TABLE IV. STUDENT QUESTIONNAIRE FEEDBACK

\begin{tabular}{lc}
\hline \hline Question & Average \\
(1= very poor, 2 = poor, $3=$ satisfactory, $4=$ good, 5 = excellent) & 3.8 \\
\hline How would you rate the OpenEEG hardware and the resources on & \\
the OpenEEG website? & 4.3 \\
\hline How would you rate the usefulness of additional resources (e.g., the & 3.6 \\
example C\#, VB and MATLAB code provided) & 4.5 \\
\hline How would you rate the usefulness of the project blog? & \\
\hline How would you rate the potential for future final-year EEG project? \\
"a very interesting project" "an exciting field" \\
What were the best aspects of your EEG project? \\
"availability of very interesting research papers" \\
"Use of MATLAB" \\
"The interactive design" ... "interesting subject, meaningful outcome" \\
\hline What content of your course, if any, was the project relevant to and did it help reinforce or \\
build upon these? \\
"Signal processing skills and concepts such as FFT, spectral power and filtering were \\
definitely reinforced". \\
"exposure to research work", "programming", "artificial intelligence". \\
\hline What were the best aspects of using the open source OpenEEG hardware and resources? \\
"The hardware is very easy to use and very cheap". \\
"We had a mini-instrument that... we were able to work with at any time" \\
"It's really easy to setup and start working, plenty of resources online". \\
\hline In terms of resourcing the project, what would you change? \\
"well resourced" \\
"Perhaps have more than one type of hardware for testing and comparing results." \\
"Information about EEG in detail" and "perhaps students could be encouraged to take ... [a \\
supporting] Module Outside Main Discipline" \\
\hline \hline
\end{tabular}

Compared to year cohorts there are, necessarily, much smaller numbers of individual project students working on a related theme with the same hardware. While it is inappropriate to draw significant conclusions from small numbers, the project outcomes and questionnaire feedback did provide some interesting insights as well as a measure of support for OpenEEG use in final-year projects.

Interesting questionnaire feedback suggestions included encouraging students toward relevant modules, and potentially modules outside main discipline which might, for example, be relevant, to EEG, neurology or physiology. Also, it had been assumed that the link with fundamental course material such as signal processing, computer interfacing, embedded systems etc. was clear, but one of the students felt that the project did not draw on prior work. This suggests that it may be useful for some students if such connections were explicitly made. In general, the feedback highlighted that students liked the potential of the EEG project theme and generally found the additional code resources beneficial. The project blog was useful, but not significantly so. The less satisfactory aspects included 
difficulties with extracting signals, e.g., system reliability, mounting electrodes and comparing signals with known test signals. The connection to research and availability of relevant publications was seen as particularly good and the availability of the equipment was very good.

\section{DISCUSSION}

One of the supervision challenges of the project was the provision of sufficient supporting resources to help students begin to develop their own applications within the time constraints of their final-year project. In the absence of platform restrictions, these resources needed to support students working on a variety of software platforms hence the development of libraries and sample code for C\#, Visual Basic and MATLAB. A challenge during the development of these resources was ambiguities in documentation accompanying the Modular OpenEEG hardware. For example, determining which firmware version and data frame format were implemented.

The Modular OpenEEG systems required regular maintenance and checking for loose connections. After 2 years of use, all boards were still functional but it was felt that the equipment would benefit from ruggedization if it were to remain useful in the longer term. The addition of active electrodes and headsets was thought to be useful.

For projects where more sophisticated signal analysis is required, EEGLAB was found to be accessible and easy-to-use. EEGLAB is a popular, well-supported open source MATLAB toolbox which provides a range of tools for processing and visualizing EEG signals [24]. The documentation generated by the EEGLAB community is extensive and includes tutorial presentations from annual international workshops.

A challenging project with a more hardware or firmware component, might involve the design a complete platform with perhaps an Arduino or PIC32 as a self-contained embedded system or with either USB output to a PC or portable computing device.

\section{CONCLUSIONS}

The use of EEG for electronic and computer engineering undergraduate projects has been shown to have a broad scope. Students have good engagement with applications for brain-computer interfacing, for gaming and for health. The projects provided practical applications of curriculum content, particularly, computer interfacing, signal processing and software design. The projects also provide an opportunity to connect with technology and applications of relevance to current research, of which there is a growing body of relevant open access literature, particularly, for example, in rehabilitation and assistive technology. This paper has outlined the projects, the challenges and some potential directions for future projects. While these projects did require additional initial support, it was felt that this was a worthwhile investment

\section{REFERENCES}

[1] OpenEEG open source EEG project on-line at http://openeeg.sourceforge.net/doc/

[2] Macy, A.J. "Students as signal sources in the biomedical engineering", in Proc. 23rd Annual International Conference of the IEEE Engineering in Medicine and Biology Society, vol. 4, pp. 4040-4043, 2001.

[3] Rhodes, S.S. and Dunne, B.E., "A hands-on bioelectric potentials course for electrical engineering majors", in Proc. 39th IEEE Frontiers in Education Conference, pp. 1-6, Oct. 2009

[4] V. Lee, et al "A mixed-signal EEG interface circuit for use in first year electronics courses" in Proc. IEEE Int. Symp. on Circuits and Systems, pp. 2689-2692, May 2012.

[5] G. R. Scolaro, F. M. Azevedo, J. E. Rathke, P. Possa, E. Andrighetto, R. Adur, J. Marino Neto,, "Development of a didactic platform for acquisition and processing of biomedical signals for the 
practice in biomedical engineering", in Proc $9^{\text {th }}$ IEEE Int. Conf. on Electronic Measurement \& Instruments, pp. 1-4, Aug. 2009.

[6] A. Jain, K. Insoo, B.J. Gluckman, 'Low cost electroencephalographic acquisition amplifier to serve as teaching and research tool', in Proc. IEEE Int. Conf. Engineering in Medicine and Biology Society, 30, pp. 1888-1891, Aug.-Sept. 2011.

[7] Lytras, M. D. and Scacchi, W., "Open-source software for engineering education: Pedagogical strategies that leverage open-source tools", IEEE Trans Education, vol. 50, no. 4, pp. 290-291., 2007.

[8] Alfonsi, B., "Open source in the classroom", IEEE Distributed Systems Online, vol. 6, no. 6, 2005.

[9] A.Palke, "Brainathlon: Enhancing brainwave control through brain-controlled game play", Master's thesis, Mills College, 2004, on-line at http://www.webkitchen.com/brainathlon/

[10] C. Veigl, "BrainBay - An open source software for biosignal and camera-mouse applications" International Conference on Computers Helping People with Special Needs (ICCHP) - Young Researchers Consortium 2006, Linz, Austria, on-line at http://www.shifz.org/brainbay/

[11] E. Niedermeyer, F. L. da Silva. Electroencephalography: Basic Principles, Clinical Applications, and Related Fields. (5th ed.), Philadelphia, PA: Lippincott Williams \& Wilkins, 2005, pp.139-141.

[12] L. A. Farwel and E. Donchin, "Talking off the top of your head: toward a mental prosthesis utilizing event-related brain potentials", Electroencephalography and Clinical Neurophysiology, vol. 70, no. 6. pp. 510-523, Dec. 1988.

[13] C. Guger, et al "How many people are able to control a P300-based-brain-computer interface (BCl)?" Neuroscience Letters, vol. 462, no. 1, pp. 94-98., 2009.

[14] D. J. Krusienski, E. W. Sellers, F. Cabestaing, S. Bayoudh, D. J. McFarland, T. M Vaughan and J. R. Wolpaw, "A comparison of classification techniques for the P300 Speller", Journal of Neural Engineering, vol. 3, no. 4, pp. 299-305, 2006.

[15] M. Thulasidas, C. Guan, J. Wu, "Robust classification of EEG signal for brain-computer interface", IEEE Trans. Neural Syst. Rehabil. Eng.Trans. , vol. 14, no. 1, pp. 24-29, 2006.

[16] J. I. Münßinger, S. Halder, S. C. Kleih, A. Furdea,V. Raco, A. Hösle, and A. Kübler, "Brain painting: first evaluation of a new brain-computer interface application with ALS-patients and healthy volunteers", Frontiers in Neuroscience, 4, 182, pp1-11, Nov. 2010.

[17] B. Rebsamen, C. Guan, H. Zhang, C. Wang, C. Teo, M. H. Ang, E. Burdet, "A brain controlled wheelchair to navigate in familiar environments", IEEE Trans. Neural Syst. Rehabil. Eng., vol.18, no. 6, pp. 590-598, Dec. 2010.

[18] Dropbox Inc, https://www.dropbox.com

[19] Olimex EEG products on-line https://www.olimex.com/Products/EEG/

[20] MATLAB, The MathWorks Inc., 2013, www.mathworks.com

[21] B.Houston, "Exocortex," 2012 [Online].

[22] Deiber, M.P. Ibanez, V. Honda, M. Sadato, N. Raman, R \& Hallett, M. - "Cerebral processes related to visuomotor Imagery and generation of simple finger movements studied with positron emission tomography," Neurolmage, vol. 7, pp. 73-85, 1998.

[23] U. Halsband, N. Ito, J. Tanji, and H-J. Freund, "The role of premotor cortex and the supplementary motor area in the temporal control of movement in man", Brain, vol. 116, no. 1, pp. 243-266, 1993.

[24] A. Delorme and S. Makeig, "EEGLAB: An open source toolbox for analysis of single-trial EEG dynamics including independent component analysis," J. Neurosci. Meth., pp. 9-21, Mar. 2004. 
T.Collins and S.I.Woolley are with the School of Electronic, Electrical and Systems Engineering, University of Birmingham, B15 2TT UK (email T.Collins@bham.ac.uk; S.I.Woolley@bham.ac.uk). N.Rawson is with Aero Engine Controls, UK and L.Haroon is with Renishaw, UK.

Tim Collins has a Ph.D. degree in Electronic and Electrical Engineering from The University of Birmingham, U.K., in 1997. He has lectured students in analog and digital electronics, and music technology. His current research interests are in signal processing for audio, health and cultural heritage applications.

Sandra Woolley has a Ph.D. degree in electronic engineering from The University of Manchester, U.K., in 1994. She is a lecturer in the School of Electronic, Electrical and Systems Engineering at The University of Birmingham, U.K. She has lectured students in a variety of subjects, including microcontrollers, multimedia and computer networking. Her current research interests include aspects of e-health and, in particular, new applications in rehabilitation and assistive technology. She also works on multidisciplinary research in cultural heritage.

Nicholas Rawson studied for his M.Eng. degree in the School of Electronic, Electrical and Systems Engineering at The University of Birmingham, U.K. His final-year M.Eng. project, entitled Sensing the Motor Cortex, is outlined in this paper. He is currently employed by Aero Engine Controls as a mechanical test engineer, designing fuel test facilities for aircraft engine components.

Luth Haroon studied for his B.Eng. and M.Sc degrees in the School of Electronic, Electrical and Systems Engineering at The University of Birmingham, U.K. He is currently employed by Renishaw in the U.K. Luth's B.Eng. final-year project, entitled Neurofeedback as a Tool for Psychoacoustical Research, is outlined in this paper. 